\title{
Arañas: tejiendo un eslabón crucial para el equilibrio de los agroecosistemas
}

\author{
Luis Guillermo Quijano-Cuervo, Luis Eduardo Robledo-Ospina, \\ Luis Fernando García Hernández y Federico Escobar Sarria
}

\begin{abstract}
Resumen
Mientras el cine, la literatura y los mitos han contribuido a la percepción errónea de las arañas como seres siniestros que habitan en lugares lúgubres y oscuros, nuestra convivencia con ellas y la importancia que tienen para la salud de los ecosistemas pasan desapercibidas. Además de describir a estos maravillosos seres ancestrales y su relación con el ser humano, en este trabajo queremos resaltar el importante papel que desempeñan las arañas como depredadores en los ecosistemas terrestres y cómo se amenaza el delicado equilibrio que estos organismos sostienen en sus telas con algunas de las prácticas agrícolas actuales.
\end{abstract}

Palabras clave: depredadores, agroecosistemas, organismos plaga, control biológico.

\section{SPIDERS: WEAVING A CRUCIAL LINK IN THE BALANCE OF AGROECOSYSTEMS}

\begin{abstract}
Movies, literature, and traditional myths created misconceptions of spiders as sinister animals living in dark and gloomy places. However, our coexistence with these organisms and their importance in the ecosystems were traditionally neglected. Thus, we want to describe these wonderful and ancient animals and their relationship with human well-being and highlight the crucial role they have in the ecosystems as predators and how agricultural practices are menacing the delicate balance that these organicisms hold with their webs.
\end{abstract}

Keywords: predators, agroecosystems, pest species, biological control. 


\section{Luis Guillermo Quijano-Cuervo}

luisquijanocuervo@gmail.com

Biólogo de la Universidad del Atlántico (Barranquilla, Colombia). Maestro en ciencias del Instituto de Ecología A.C. (INECOL) donde actualmente es estudiante de doctorado. Sus intereses de investigación incluyen el estudio de la ecología y taxonomía de arañas. Específicamente sus investigaciones están enfocadas en entender cómo las características del paisaje modulan la diversidad de arañas. Además, le interesa descifrar el papel depredador de las arañas en los agroecosistemas a través de enfoques espacialmente explícitos, análisis de material genético y experimentos controlados.

\section{Luis E. Robledo-Ospina}

luis.robledo86@yahoo.es

Biólogo de la Universidad de Caldas (Manizales), Magister en ciencias por el Instituto de Ecología A.C. (INECOL) de Xalapa, México, donde actualmente realiza estudios de doctorado. Pertenece al Grupo de Aracnología de la Universidad de Caldas (GAUc), Manizales, Colombia. Sus intereses de investigación incluyen el estudio de señales visuales en arañas, dirigidas a evitar la depredación o comunicarse con sus conespecíficos, y las implicaciones de estas señales en la ecología y evolución de las arañas.

\section{Luis Fernando García Hernández}

luizf.garciah@gmail.com

Biólogo de la Universidad Militar Nueva Granada (Colombia), Magister y Doctor en ciencias biológicas (Zoología) por el Programa para el Desarrollo de las Ciencias Básicas (PEDECIBA) de la Universidad de la República del Uruguay. Actualmente se desempeña como docente en el Centro Universitario Regional del Este (CURE) en Uruguay. Pertenece al Sistema Nacional de Investigadores (SNI) del Uruguay y es Investigador en los programas de posgrado de Ciencias Básicas (zoología) y de la Facultad de Agronomía de la Universidad de la República. Sus intereses de investigación se centran en la ecología trófica de arácnidos y en la aplicación de estos conceptos en las especies presentes en sistemas agrícolas. De igual manera, sus estudios engloban a la biología y a la diversidad de especies poco conocidas de arácnidos.

\section{Federico Escobar Sarria}

federico.escobar@inecol.mx

Investigador Titular en el Instituto de Ecología A.C. (INECOL), e Investigador Nacional Nivel II del Sistema Nacional de Investigadores Consejo Nacional de Ciencia y Tecnología, conacyT. Sus intereses de investigación incluyen los estudios de ecología de comunidades y los estudios de diversidad, principalmente en escarabajos coprófagos. Adicionalmente, ha realizado diversos estudios en agroecosistemas, evaluando la relación entre la biodiversidad y la actividad productiva en esos ambientes. 


\section{Las arañas y los humanos}

Cuando pensamos en bichos, alimañas y otras criaturas que habitan la tierra, es común que las arañas sean confundidas con sus parientes cercanos, los insectos, ya que ambos están cercanamente emparentados (podemos decir que son primos lejanos) y es por eso que comparten varias características, una de ellas es la presencia de un esqueleto externo, además de sus patas y de sus otros apéndices articulados, que le permite desplazarse y manipular su alimento, los biólogos las clasificaron como artrópodos (Arthropoda, del griego àpӨpov, árthron, "articulación” y пoúc, poús, "pie”).

Las arañas pertenecen a un grupo particular de artrópodos terrestres llamados arácnidos (Arachnida), que además agrupan a escorpiones (comúnmente llamados alacranes), los opiliones o cosecheros y otros grupos menos conocidos como los seudoescorpiones, amblipigios (o arañas patonas), entre otras especies. Estos organismos se distinguen por la ausencia de antenas y alas, por presentar cuatro pares de patas, el cuerpo dividido en dos segmentos, y por tener un par de apéndices bucales llamados quelíceros y otro par llamados pedipalpos, cuya forma y función son variables, pero ambos son estructuras claves para la alimentación y la reproducción de las arañas.

Las arañas forman parte importante de la cultura de distintas civilizaciones, se tienen registros muy antiguos sobre su existencia, estos se remontan a más de 5000 años. Debido a diversas razones, se les ha asignado un papel ambivalente: mientras que en algunas culturas, las arañas representan la muerte y la destrucción, en otras, son símbolos de fertilidad, de protección, de creación y de vida (Melic, 2002). No obstante, para entender el porqué de esta dualidad, es necesario conocer algunos de sus rasgos característicos que las destacan como seres claves en la naturaleza y así, como consecuencia, poder acabar con su mala fama.

Como primer rasgo se debe entender que debido a su condición de depredadores estrictos (es decir, que se alimentan de otros animales), la mayoría de las especies de arañas presentan glándulas venenosas asociadas a los quelíceros, que tienen como función paralizar y predigerir a sus presas, generalmente insectos y otros pequeños artrópodos. Tal vez por esta razón y debido a los mecanismos primordiales para la detección de animales peligrosos dentro de las sociedades, las arañas, tradicionalmente, han sido reconocidas como una amenaza para la supervivencia humana. (New et al., 2015). No obstante, la realidad es que de las más de 49 mil especies descubiertas actualmente (World Spider Catalog 2021), menos de 30 especies representan una amenaza real para los humanos y, por el contrario, como veremos enseguida, son más los beneficios que nos brindan estos diminutos, delicados y maravillosos seres.

Sin lugar a duda, el rasgo más distintivo de las arañas es la producción de seda a través de una serie de glándulas ubicadas en su abdomen. La seda de las 
Imagen 1. Diosa maya |x-Chel, del amor, la gestación, el agua, la luna, la medicina y los trabajos textiles. Cortesía de Thalia Took. arañas es una fibra compuesta por proteínas llamadas espidroínas, y la ocupan en diversas tareas, como en la construcción de refugios, en la captura y almacenamiento de presas, en la protección de sus huevos, entre otras. Según esto, podemos considerar a la seda de las arañas como una extensión de su propio cuerpo. Un ejemplo de su representación benévola lo encontramos en la cultura Maya, donde la telaraña representa la placenta de Ix-Chel, diosa de la luna, del parto y patrona de las tejedoras (ver imagen 1).

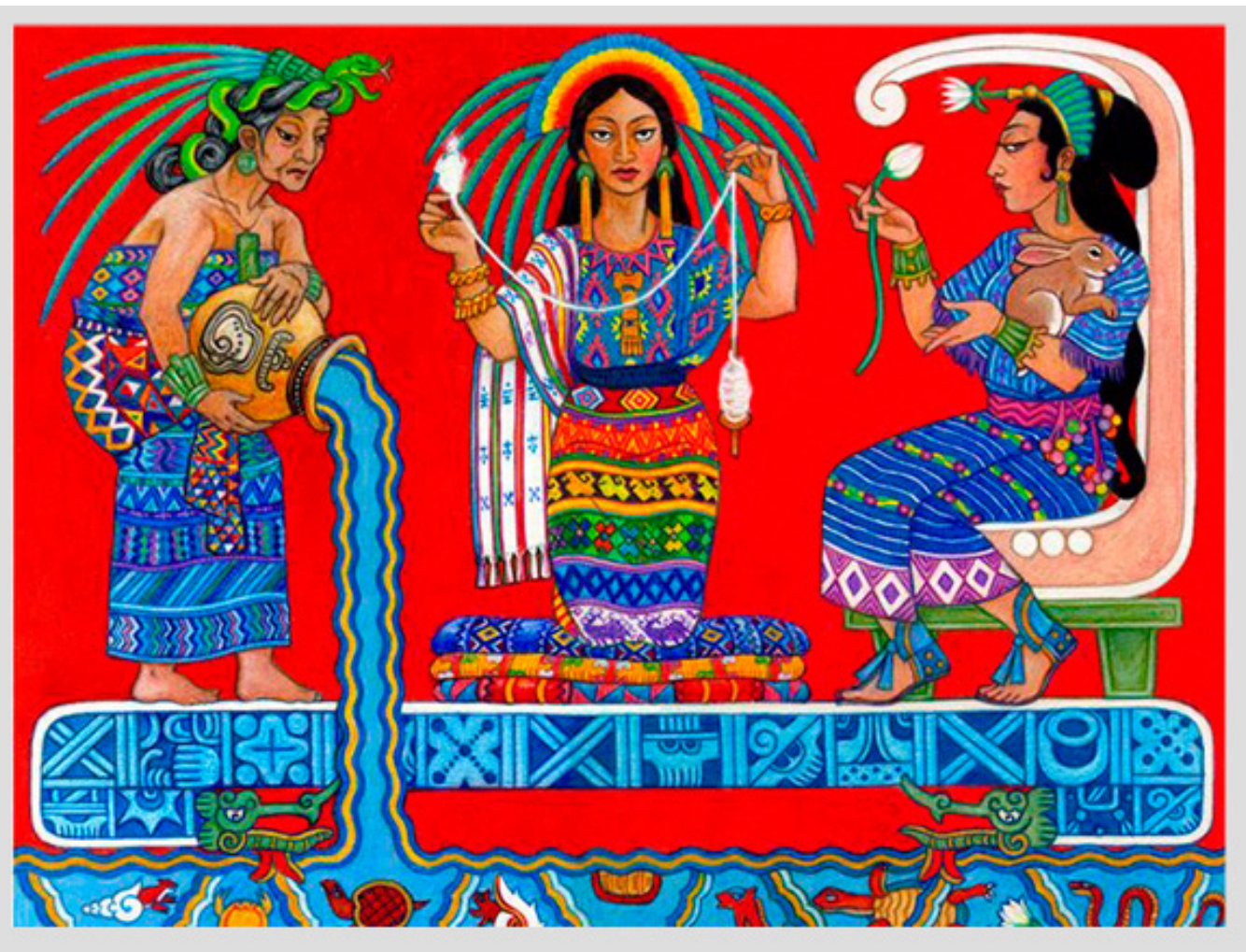

\section{Las arañas: Ios mayores depredadores en los ecosistemas terrestres}

Desde su aparición hace casi 380 millones de años, las arañas representan uno de los grupos de animales terrestres más diversos sobre la tierra, lo que se refleja en su amplia variedad de formas, tamaños y colores. Abarcan casi todos los ecosistemas, desde bosques tropicales de tierras bajas hasta bosques fríos en las montañas, zonas secas y desiertos. Un dato interesante es que representan una biomasa global estimada en más de 25 millones de toneladas (masa corporal estimada de todas las arañas del planeta), por lo que se calcula que las arañas pueden consumir entre 400 y 800 millones de toneladas de alimento anualmente (Nyffeler et al., 2017), ¡lo equivalente a 4 mil y 8 mil veces el peso del Estadio Azteca! Esto las convierte en los depredadores más voraces y eficaces del planeta. 
Teniendo en cuenta que la mayoría de las especies de arañas se alimentan de muchos tipos de insectos, ellas desempeñan un papel clave como controladoras naturales de plagas y de vectores de enfermedades. Por lo anterior, las arañas contribuyen al mantenimiento del delicado equilibrio de los ecosistemas naturales y de aquellos derivados de la actividad humana, en particular de los cultivos, de los cuales dependemos para nuestra alimentación.

El éxito de las arañas como depredadoras se debe, en gran medida, a la amplia cantidad de estrategias de cacería que han desarrollado a lo largo de su historia evolutiva (Cardoso et al., 2011). Por ejemplo, aunque todas las arañas tienen la capacidad de producir telaraña, no todas la usan para cazar y solo algunas especies construyen redes aéreas para interceptar a sus presas en vuelo, las arañas tejedoras, por ejemplo. Mientras que las arañas emboscadoras se camuflan antes de atacar a la presa. También existen especies que cazan activamente, conocidas como arañas acechadoras, que se esconden y esperan a la presa, aproximándose sigilosamente y abalanzándose sobre ella, muy similar a como lo hacen los felinos. Incluso, algunas especies de arañas son capaces de capturar renacuajos y pequeños peces debajo del agua.

Imagen 2. Telaraña orbicular. La telaraña orbicular es un tipo de telaraña aérea que se menciona en el párrafo inmediatamente anterior, por lo tanto podría dejarse simplemente como telaraña, para evitar confundir al lector. Foto tomada por Kevin Dorian Tinoco Barrera en Pátzcuaro, Michoacán, México.

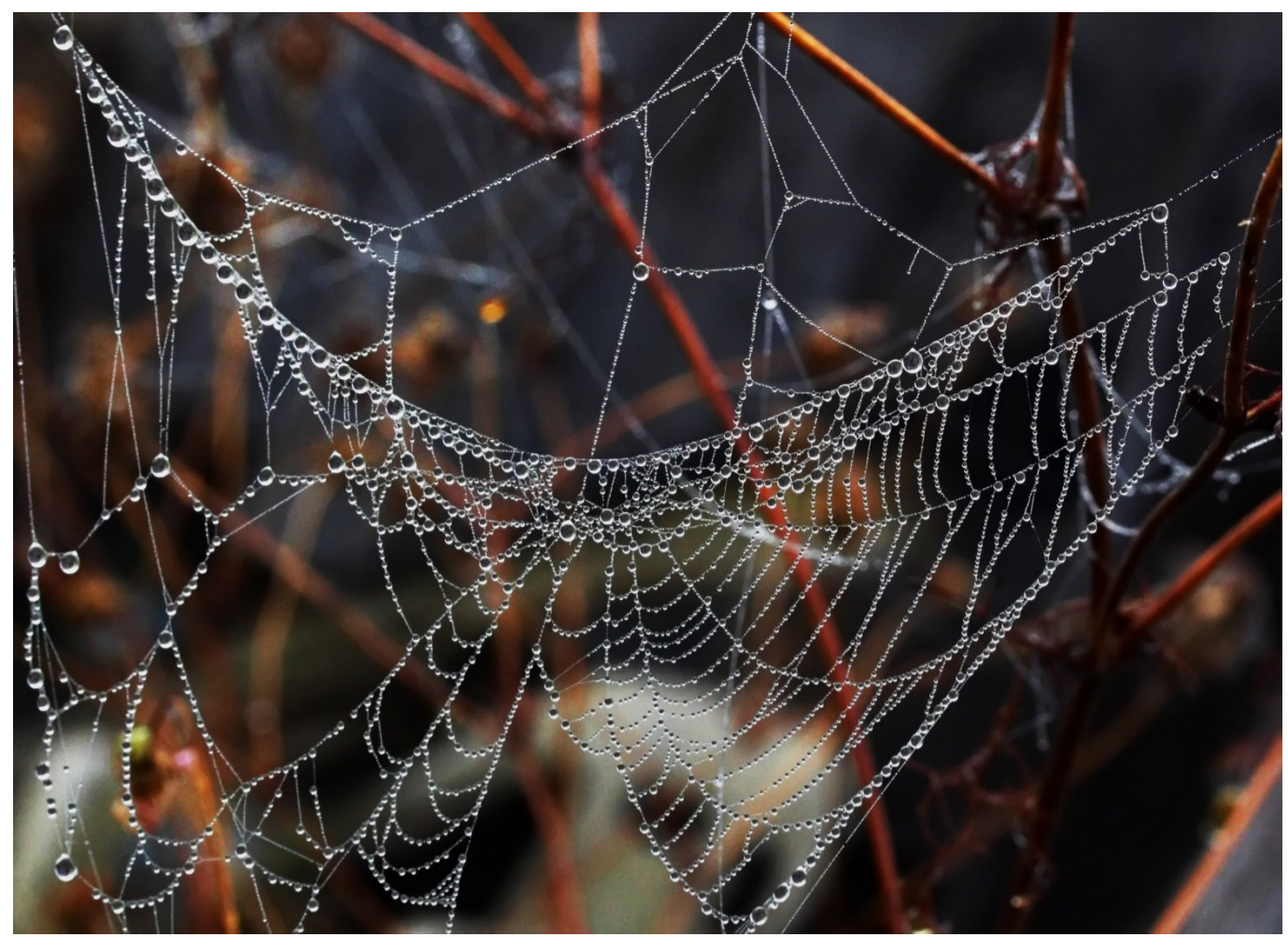

\section{Las arañas y los agroecosistemas}

La evolución de la especie humana y su desarrollo cultural ha modificado los ecosistemas naturales. Además, el paso del Homo sapiens: de cazador nómada a domesticador y trabajador de la tierra, dio como resultado la transformación 
Imagen 3. Araña lobo (Lycosidae) depredando a la polilla guatemalteca de la papa, Tecia solanivora Povolni 1973 (Lepidoptera: Gelechiidae) Créditos: CInstituto ENTOMANaturavisión de los bosques y la aparición de un nuevo tipo de paisaje en el que dominan los cultivos de pocas especies de plantas (monocultivos) y la presencia de animales domésticos. Estos nuevos ecosistemas, también conocidos como agroecosistemas, a menudo entran en conflicto con la conservación de los bosques y de los recursos naturales, lo cual genera desequilibrios ecológicos debido a la implementación de prácticas de manejo poco sustentables como el uso excesivo de productos agroquímicos, los cuales tienen efectos nocivos para la salud humana.

Como contrapartida al manejo tradicional de los agroecosistemas, el control biológico conservativo surge como una estrategia basada en el aprovechamiento de los enemigos naturales autóctonos (propios del ecosistema) de un determinado cultivo para mantener las plagas al margen. Dentro de los depredadores nativos que podemos encontrar en una amplia variedad de sistemas agrícolas, las arañas son uno de los grupos más abundantes y diversos. Su presencia en los agroecosistemas puede traer beneficios a los productores debido a que estas tienen la capacidad de disminuir las poblaciones de insectos consumidores de las hojas, las flores y los tallos de los cultivos (conocidos como insectos herbívoros o fitófagos), lo cual reduce el daño a las plantas e incrementa la productividad de los sistemas agrícolas (Michalko et al., 2019a, 2019b, ver imagen 3).

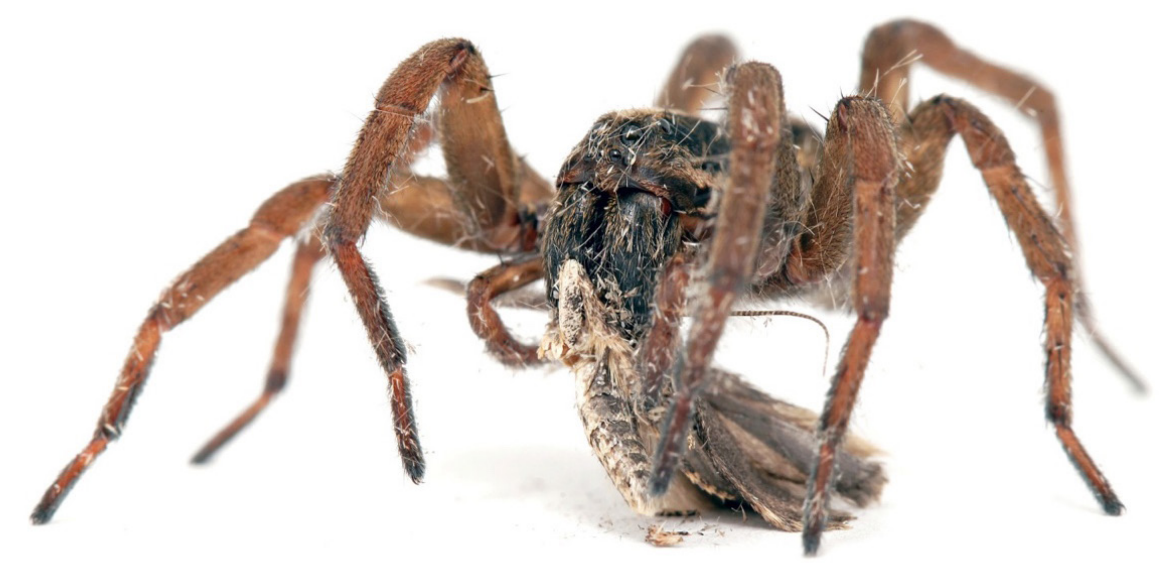

Indirectamente, las arañas también pueden disminuir el impacto de los insectos herbívoros en los cultivos, ya que algunas dejan rastros químicos (como la seda) sobre las plantas, lo cual es tomado como una advertencia por parte de los insectos (Michalko et al., 2019b), esto es conocido como efectos de no consumo. Sin embargo, a pesar de la importancia de las arañas como depredadoras, los estudios sobre ellas en los sistemas agrícolas, particularmente en Latinoamérica, son escasos, y como consecuencia, su papel dentro de estos sistemas es subestimado (Benamú et al., 2017a). Esto es particularmente importante en los programas de control biológico conservativo, los cuales se sugieren como la base de los programas de control biológico que se implementan en la actualidad, ya que resultan benéficos para la conservación de la fauna y flora autóctona (van Lenteren et al., 2018). 
Las prácticas convencionales, empleadas habitualmente en los cultivos, pueden interferir con los servicios ecosistémicos que nos aportan las arañas. Una de las prácticas más letales para las arañas es el uso excesivo de agroquímicos ya que, si no las mata, muchas de las sustancias químicas usadas pueden modificar su comportamiento y su capacidad de reproducción (Pekár, 2012). A pesar de que el uso de agroquímicos en los cultivos no tiene como finalidad reducir las poblaciones de arañas, el desconocimiento sobre sus efectos secundarios los convierte en una práctica que genera efectos negativos sobre este grupo de depredadores inmersos en los agroecosistemas.

En relación con lo anterior, estudios recientes en América Latina demuestran que los agroquímicos pueden modificar las propiedades fisicoquímicas de la seda que producen las arañas (Benamú et al., 2017b) y la construcción de sus telarañas (Benamú et al., 2010). Adicionalmente, otros estudios realizados en esta misma región indican que los agroquímicos como el glifosato (un agroquímico de uso común) pueden reducir la capacidad y la efectividad de las arañas para consumir presas en un determinado tiempo debido a que genera un efecto irritante en ellas (Lacava et al., 2021).

Además, si calculáramos el costo monetario del papel que cumplen las arañas como depredadores en los ecosistemas terrestres, sería evidente el impacto negativo para los humanos. Por ejemplo, en el año de 1994 se estimó que el servicio de control biológico que prestaron ciertos depredadores claves, como las arañas, en los agroecosistemas, fue de más de 33 mil millones de dólares por año (Costanza et al., 1997). Debido a esto, es realmente difícil pensar en reemplazar la labor que desempeñan estos organismos depredadores con métodos de control no biológicos.

Otro aspecto de suma importancia para la conservación de la diversidad de las arañas en los agroecosistemas está relacionado con la forma en cómo los agricultores pueden favorecer la variedad vegetal dentro y alrededor de los cultivos. Por ejemplo, pueden mantener una mayor diversidad vegetal dentro y alrededor de los cultivos para generar más sitios que puedan ser colonizados por las arañas y como consecuencia, incrementar su número de especies e individuos. Esta diversidad vegetal se puede lograr a través de policultivos, los cuales son sistemas que producen simultáneamente cultivos de diferentes productos en una misma zona, un ejemplo de esto son las milpas, los cafetales y cacaotales de sombra; o también, esto se puede lograr si se mantienen remanentes de vegetación nativa aledaños a los cultivos que permitan el movimiento entre ambientes, tanto de las poblaciones de arañas, como de otras especies de las cuales se alimentan.

\section{El estudio de las arañas en los cultivos de México}

México se caracteriza por ser uno de los principales productores de diversos cultivos como lo son, el maíz, el aguacate, el café y el chile. No obstante, uno de 
Imagen 4. Arañas de las familias Oxyopidae (izquierda) y Araneidae (derecha). Estas familias junto con Salticidae y Tetraghnatidae son de las más comunes en sistémas agrícolas. Las fotos particularmente se tomaron en ese tipo de ecosistemas en Pátzcuaro Foto tomada por Kevin Dorian Tinoco Barrera en Pátzcuaro, Michoacán, México. los mayores problemas, en varios de estos sistemas agrícolas, son las enormes pérdidas económicas provocadas por plagas, en su mayoría por invertebrados, como moscas, larvas de escarabajos y mariposas (por ejemplo, el cogollero del maíz Spodoptera frugiperda, una de las plagas más importantes del continente americano). El control natural de los organismos plaga siempre se ha visto como una alternativa más amigable que el uso desmedido de insecticidas. Sin embargo, debido al poco conocimiento de la ecología y del comportamiento de los depredadores naturales, como las arañas, el control biológico realizado por los depredadores nativos es una alternativa, hasta ahora, poco explorada en el país.

Los estudios de arañas en los cultivos de México son escasos, y en su mayoría se enfocan a realizar un inventario de las especies presentes en los cultivos como el café y el cacao (ver Pinkus-Rendón et al., 2006). Muy pocas investigaciones son las que se centran en demostrar el papel de los cultivos orgánicos para la conservación de las arañas. Por lo anterior, el conocimiento y el estudio sobre el papel que desempeñan las arañas en los agroecosistemas de México aún es insuficiente, por lo que exhortamos a la comunidad científica y a futuras generaciones al estudio de estos importantes depredadores. Creemos que, con el conocimiento adecuado de la biología y del comportamiento de las arañas, se logrará consolidar estrategias de manejo que contribuyan a mitigar los daños causados por plagas en los cultivos y se ayudará a reducir la necesidad de usar agroquímicos sin afectar la economía de los agricultores mexicanos, mientras que al mismo tiempo se conservan las comunidades naturales de artrópodos que forman parte importante del equilibrio ecológico.

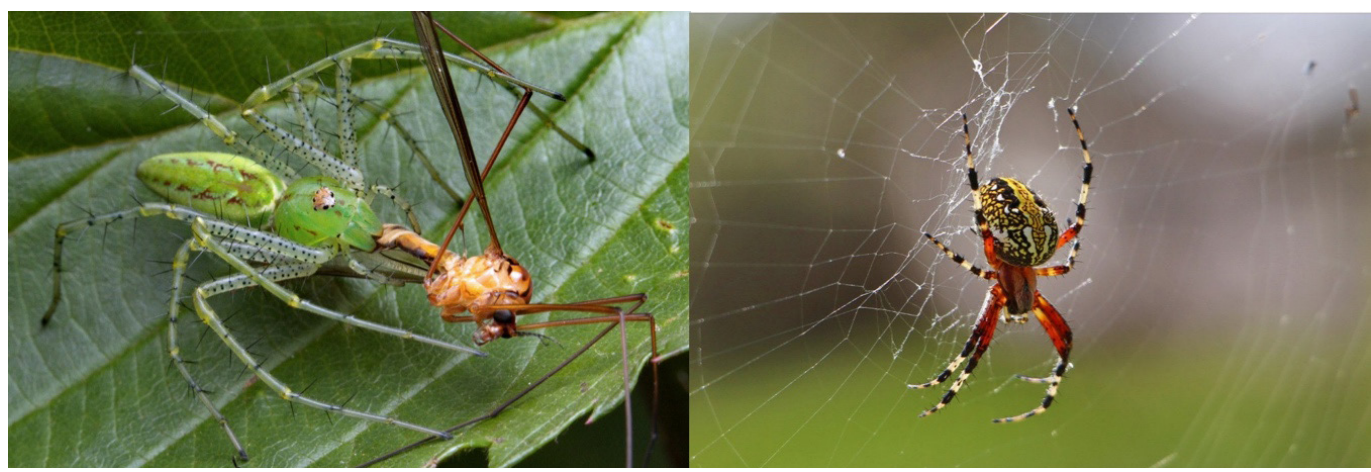

\section{Recomendaciones finales para tratar a las arañas en los cultivos y para los cultivadores}

Finalmente, desde la ecología y desde el conocimiento biológico de este grupo de depredadores, es importante considerar algunas recomendaciones que pueden ayudar a favorecer comunidades de arañas saludables en los cultivos, sin antes, recordar lo más importante: todos podemos coexistir en este planeta, siempre y cuando velemos por el equilibrio y la conservación de la vida. 
1. Evitar el uso desmedido de pesticidas.

2. Tener en cuenta que las arañas no son plagas, aunque pueden alcanzar grandes densidades dentro de los cultivos.

3. Evitar, en lo posible, simplificar los sistemas con monocultivos.

4. Tener cerca de los cultivos algunas fuentes de comunidades saludables de arañas, como bosques, zonas en regeneración, cercas vivas y vegetación ribereña.

\section{Referencias}

* Benamú, M. A., Schneider, M. I. y Sánchez, N. E. (2010). Effects of the herbicide glyphosate on biological attributes of Alpaida veniliae (Araneae, Araneidae), in laboratory. Chemosphere, 78(7), 871-876. https://doi.org/10.1016/j. chemosphere.2009.11.027

* Benamú, M. A., Lacava, M., García, L. F., Santana, M. y Viera, C. (2017a). En C. Viera y M. Gonzaga (Eds.). Chapter 11. Spiders associated with agroecosystems: Roles and perspectives. Behaviour and Ecology of Spiders: Contributions from the Neotropical Region (pp. 275-302). Springer International Publishing. https://doi. org/10.1007/978-3-319-65717-2

* Benamú, M. A., Lacava, M., García, L. F., Santana, M., Fang, J., Wang, X. y Blamires, S. J. (2017b, agosto). Nanostructural and mechanical property changes to spider silk as a consequence of insecticide exposure. Chemosphere, 181, 241-249. https:// doi.org/10.1016/j.chemosphere.2017.04.079

* Cardoso, P., Pekár, S., Jocqué, R. \& Coddington, J. A. (2011, 29 de junio). Global patterns of guild composition and functional diversity of spiders. PLOS ONE, 6(6), e21710. https://doi.org/10.1371/journal.pone.0021710

* Costanza, R., d'Arge, R., de Groot, R., Stephen, F., Grasso, M., Hannon, B., Limburg, K., Naeem, S., O'Neill, R. V., Paruelo, J., Raskin, R. J., Sutton, P. y van den Belt, M. (1997). The value of the world's ecosystem services and natural capital. Nature, 387, 253-260. https://doi.org/10.1038/387253a0

- Lacava, M., García, L. F., Viera, C. y Michalko, R. (2021, enero). The pest-specific effects of glyphosate on functional response of a wolf spider. Chemosphere, 262, 127785. https://doi.org/10.1016/j.chemosphere.2020.127785

* Melic, A. (2002). De Madre Araña a demonio Escorpión: Los arácnidos en la Mitología. ARACNet 10 - Revista Ibérica de Aracnología (Boletín), 5(2002): 112-124. http://entomologia.rediris.es/aracnet/e2/10/03mitologia/

* Michalko, R., Pekár, S., Dul'a, M. y Entling, M. H. (2019a). Global patterns in the biocontrol efficacy of spiders: A metaDanalysis. Global Ecology and Biogeography, 28, 1366-1378. https://doi.org/10.1111/geb.12927

* Michalko, R., Pekár, S. y Entling, M. H. (2019b). An updated perspective on spiders as generalist predators in biological control. Oecologia, 189, 21-36. https://doi. org/10.1007/s00442-018-4313-1 
* New, J. J, y German, T. C. (2015). Spiders at the cocktail party: an ancestral threat that surmounts inattentional blindness. Evolution and Human Behavior, 36(3), 165173. https://doi.org/10.1016/j.evolhumbehav.2014.08.004

* Nyffeler, M., y Birkhofer, K. (2017). An estimated 400-800 million tons of prey are annually killed by the global spider community. The Science of Nature, 104(30). https://doi.org/10.1007/s00114-017-1440-1

* Pekár S. (2012). Spiders (Araneae) in the pesticide world: an ecotoxicological review. Pest Management Science, 68(11), 1438-1446. https://doi.org/10.1002/ ps.3397

* Pinkus-Rendón, M. A., León-Cortés, J. L. e Ibarra-Núñez, G. (2006, enero). Spider diversity in a tropical habitat gradient in Chiapas, Mexico. Diversity and Distributions, 12(1), 61-69. https://doi.org/10.1111/j.1366-9516.2006.00217.x

* Took, T. (2004-2013). Ix Chel. A-Muse-ing Grace Gallery. The Magical Art of Thalia Took. http://www.thaliatook.com/AMGG/ixchel.php

van Lenteren, J. C., Bolckmans, K., Köhl, J., Ravensberg, W. J. y Urbaneja, A. (2018). Biological control using invertebrates and microorganisms: plenty of new opportunities. BioControl, 63, 39-59. https://doi.org/10.1007/s10526-017-9801-4

* World Spider Catalog. (2021). World Spider Catalog. Version 21.0. Natural History Museum Bern. http://wsc.nmbe.ch

\section{Agradecimientos}

LGQ-C y LER-O agradecen al Instituto de Ecología A.C. (INECOL) y al Consejo Nacional de Ciencia y Tecnología (CONACYT) por el apoyo para el desarrollo de sus programas de doctorado en ciencias (becas CONACYT No. 862395 y CONACYT No. 634812/338721 respectivamente). LFG agradece a la Agencia Nacional de Investigación e Innovación y a la Comisión Sectorial de Investigación Científica (csic), por el apoyo para el desarrollo de las líneas de investigación. Finalmente, agradecemos a ENTOMA-Naturavisión y Kevin Dorian Tinoco Barrera quienes amablemente facilitaron fotografías para ilustrar el texto.

\section{Cómo CITAR ESTE ARTículo}

* Quijano-Cuervo, Luis Guillermo, Robledo-Ospina, Luis Eduardo, García Hernández, Luis Fernando y Escobar Sarria, Federico. (2021, mayo-junio). Arañas: tejiendo un eslabón crucial para el equilibrio de los agroecosistemas. Revista Digital Universitaria (RDU), 22(3). Dol: http://doi.org/10.22201/cuaieed.16076079e.2021.22.3.5 